\title{
Predictive Models of Current, Voltage, and Power Losses on Electric Transmission Lines
}

\author{
O. M. Bamigbola, ${ }^{1}$ M. M. Ali, ${ }^{2}$ and K. O. Awodele ${ }^{3}$ \\ ${ }^{1}$ Department of Mathematics, University of Ilorin, Ilorin 240003, Nigeria \\ ${ }^{2}$ School of Computational and Applied Mathematics, Faculty of Science, and TCSE, Faculty of Engineering and the Built Environment, \\ University of the Witwatersrand (Wits), Private Bag 3, Johannesburg 2050, South Africa \\ ${ }^{3}$ Department of Electrical Engineering, University of Cape Town, Private Bag X3, Rondebosch, Cape Town 7701, South Africa
}

Correspondence should be addressed to M. M. Ali; montaz.ali@wits.ac.za

Received 2 January 2014; Accepted 1 February 2014; Published 5 March 2014

Academic Editor: Aderemi Oluyinka Adewumi

Copyright ( 2014 O. M. Bamigbola et al. This is an open access article distributed under the Creative Commons Attribution License, which permits unrestricted use, distribution, and reproduction in any medium, provided the original work is properly cited.

A modern and civilized society is so much dependent on the use of electrical energy because it has been the most powerful vehicle for facilitating economic, industrial, and social developments. Electrical energy produced at power stations is transmitted to load centres from where it is distributed to its consumers through the use of transmission lines run from one place to another. As a result of the physical properties of the transmission medium, some of the transmitted power is lost to the surroundings. The overall effect of power losses on the system is a reduction in the quantity of power available to the consumers. An accurate knowledge of transmission losses is hinged on the ability to correctly predict the available current and voltage along transmission lines. Therefore, mathematical physics expressions depicting the evolution of current and voltage on a typical transmission line were formulated, and derived therefrom were models to predict available current and voltage, respectively, at any point on the transmission line. The predictive models evolved as explicit expressions of the space variable and they are in close agreement with empirical data and reality.

\section{Introduction}

The importance of electric power in today's world cannot be overemphasized for it is the key energy source for industrial, commercial, and domestic activities [1]. Its availability in the right quantity is essential to the advancement of civilization.

Electrical energy is generated at power stations which are usually situated far away from load centers. As such, an extensive network of conductors between the power stations and the consumers is required. This network of conductors may be divided into two main components, called the transmission system and the distribution system. The transmission system is to deliver bulk power from power stations to load centers and large industrial consumers while the distribution system is to deliver power from substations to various consumers.

The efficiency of the transmission component of the electric power system is known to be hampered by a number of problems, especially in third-world countries. The major problems identified in [2] include application of inappropriate technology, inadequacy of materials, equipment, and man power.

From the physics of electric power transmission, when a conductor is subjected to electric power (or voltage), electric current flows in the medium. Resistance to the flow produces heat (thermal energy) which is dissipated to the surroundings. This power loss is referred to as ohmic loss [3]. Furthermore, if the applied voltage exceeds a critical level, another type of power loss, called the corona effect [4], occurs. The power losses accumulate as the induced current flows and the corona effect propagate along the transmission lines. The power losses could take off a sizeable portion of the transmitted power since transmission lines usually span a long distance, sometimes several hundred kilometers [5]. The overall effect of power losses on the system is a reduction in the quantity of power available to the consumers. Therefore, 


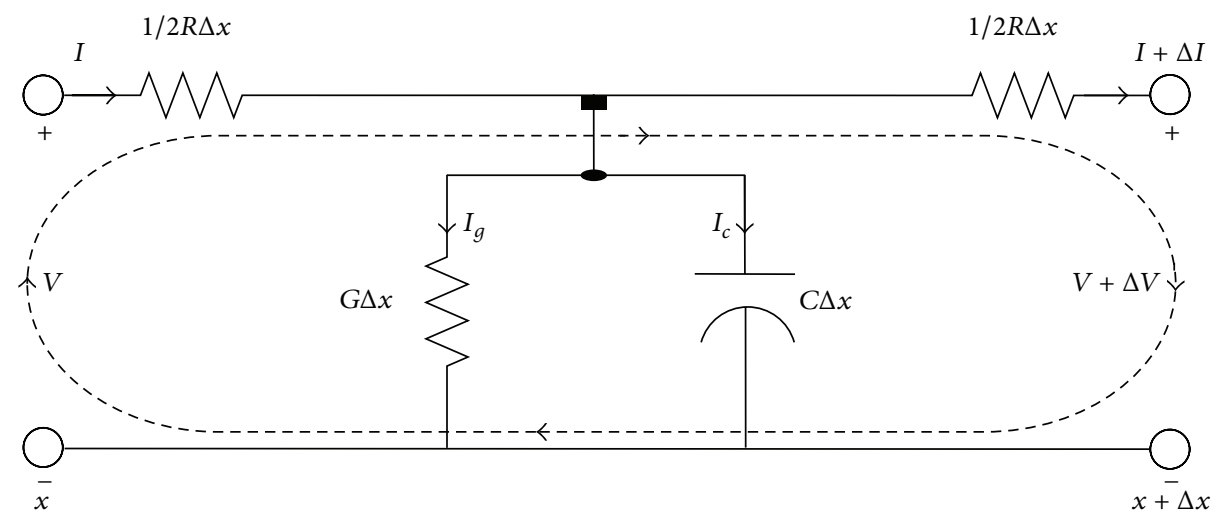

FIgURE 1: Equivalent circuit of a transmission line.

an accurate knowledge of power losses on transmission lines will be useful in planning for the supply of sufficient quantity of power needed in an electrical network.

One way of mitigating losses in the process of transmitting electric power is to apply some strategies to reduce the losses. Ramesh et al. [6] looked at minimization of power loss in distribution networks by using feeder restructuring, implementation of distributed generation, and capacitor placement method. Rugthaicharoencheep and Sirisumrannukul [7] employed the use of feeder reconfiguration for loss reduction in distribution system with distributed generators by Tabu Search. Sinsuphun et al. [8] worked on loss minimization using optimal power flow based on swarm intelligences. Recently, the classical optimization technique was applied to formulate the optimal strategy that reduces the transmission power losses to the barest minimum [9]. The strategy is to transmit electric power at very low current with high operating voltage close to the critical disruptive voltage and the spacing between the transmission lines not less than the value of $r \cdot e^{U_{p} / 18 r}$ where $r$ and $U_{p}$ are radiuses of the transmission medium and the phase voltage, respectively.

In this paper, we propose mathematical models for predicting available current and voltage as well as the power losses along a typical transmission line so as to be able to reckon the net electric power available to be used to meet customers' demands. In the process, the evolution of current and voltage on the transmission line is studied and models to predict both current and voltage were constructed. In the end, the desired model for predicting power losses along transmission lines were formulated by reframing the power loss function as a mathematical physics problem. This strategy led to the exclusion of all the transmission parameters from the model.

In the next section, we derive the equations that characterise the evolution of electric current and voltage on a typical transmission line. In Section 3, a predictive model of the power losses incurred at different locations on the transmission line is obtained. An analysis of the models is conducted and discussed in Section 4, while the paper is concluded in Section 5.

\section{Power Flow on Transmission Lines}

In this section, we derive the expressions which voltage and current must satisfy on uniform transmission lines. A real transmission line will have some series resistance associated with power losses in the conductor [10]. There may also be some shunt conductance if the insulating material holding two conductors has some leakage current. Therefore, resistance and conductance are responsible for power losses on transmission lines [11]. To this end, we formulate a model for a lossy transmission line where the effect of the series resistance $(R)$ and shunt conductance $(G)$ is taken care of on the transmission lines.

2.1. Model Formulation. Herein, we are interested in determining the extent to which voltage and current outputs differ from their input values over an elemental portion of the transmission line. As such, we consider an equivalent circuit of a transmission line of length $\Delta x$ containing resistance $R \Delta x$ and conductance $G \Delta x$ as shown in Figure 1 . The circuit illustrates how power (both voltage and current) flow through the transmission medium is considered positioned along the space variable $x$ ([12], pp. 255-260). Applying the Kirchoff voltage law [13] on the equivalent circuit of the transmission line, we have

$$
V=\frac{1}{2} R I \Delta x+\frac{1}{2} R[I+\Delta I] \Delta x+V+\Delta V,
$$

which on simplification, dividing through by $\Delta x$ and taking limits as $\Delta x$ tends to zero, is simplified to

$$
\frac{d V}{d x}=-R I
$$

and from which we have

$$
\frac{d^{2} V}{d x^{2}}=-R \frac{d I}{d x}
$$

Using the Kirchoff current law [13],

$$
I=\frac{1}{2} G\left(V+\frac{\Delta V}{2}\right) \Delta x+C \frac{d}{d t}\left(V+\frac{\Delta V}{2}\right) \Delta x+I+\Delta I,
$$


which also on simplification, dividing through by $\Delta x$ and taking limits as $\Delta x$ tends to zero, is simplified to

$$
\frac{d I}{d x}=-\left[G V+C \frac{d V}{d t}\right]
$$

which is further simplified to

$$
\frac{d I}{d x}=-G V
$$

since $V$ is independent of $t$. Differentiating (6) with respect to $x$ again results in

$$
\frac{d^{2} I}{d x^{2}}=-G \frac{d V}{d x}
$$

Substituting (6) into (3) and (2) into (7) yields

$$
\begin{aligned}
& \frac{d^{2} V}{d x^{2}}=R G V \\
& \frac{d^{2} I}{d x^{2}}=R G I .
\end{aligned}
$$

Equations (8) and (9) are mathematical physics expressions that characterise the power flow along the transmission lines.

Solving power (voltage) flow equation (8) which is subject to the boundary conditions,

$$
\begin{gathered}
V(0)=V_{0} \\
V(l)=0, \quad l \longrightarrow \infty,
\end{gathered}
$$

where $V_{0}$ is the initial voltage, results in

$$
V(x)=V_{0} e^{-x \sqrt{R G}}
$$

The equivalent solution to (9) for current flow along the transmission line is

$$
I(x)=I_{0} e^{-x \sqrt{R G}},
$$

where $I_{0}$ denotes the initial current in the circuit.

With the aid of the last two equations, the quantity of current and voltage at any point on the transmission line can be discerned. Table 1 presents the predicted current and voltage at some points on a typical $330 \mathrm{kV}$ single circuit of the Nigerian transmission network. Presently, the maximum length of the transmission network is about $300 \mathrm{~km}$.

In the next section, we seek to predict the total power losses over a typical transmission line.

\section{Power Losses on Transmission Lines}

The main reason for losses on transmission lines is the resistance of the conductor against the flow of current [14]. As a result, heat is produced in the conductor and this increases the temperature of the conductor. The rise in the conductor's temperature further increases the resistance of the conductor
TABLE 1: Current and voltage along a $330 \mathrm{kV}$ single circuit of a typical transmission network with $R G=7.15 \times 10^{-5}$.

\begin{tabular}{lcc}
\hline Length of line $(\mathrm{km})$ & Current $(\mathrm{A})$ & Voltage $(\mathrm{kV})$ \\
\hline 10 & 19.14 & 329.5 \\
20 & 19.09 & 329.1 \\
50 & 19.00 & 327.6 \\
100 & 18.87 & 325.3 \\
200 & 18.60 & 320.7 \\
300 & 18.33 & 316.1 \\
\hline
\end{tabular}

and this consequently increases the power losses. The value of the ohmic power loss [15] is given as

$$
L_{O}=I^{2} R,(\mathrm{~kW}) \text {, }
$$

where $I$ denotes current along the conductor and $R$ represents resistance of the conductor.

The formation of corona on transmission lines is associated with loss of power too, which will have some effect on the efficiency of the transmission line [16]. Corona discharge has to do with emission of ions from the surface of the transmission medium [17]. The corona power loss for a fair weather condition $[18,19]$ has the value

$$
L_{C}=242 \frac{(f+25)}{\delta} \cdot \sqrt[4]{\frac{r}{d}}\left(V-V_{c}\right)^{2} \cdot(10)^{-5} \mathrm{~kW},
$$

where $f$ represents the frequency of transmission, $\delta$ denotes the air density factor, $r$ is radius of the conductor, $d$ represents the space between the transmission lines, $V$ is the operating voltage, and $V_{c}$ denotes the disruptive voltage. High voltage gradients of above $18 \mathrm{kV} / \mathrm{cm}$ surrounding conductors is known to cause corona discharge ([20], pg. 645).

The total losses on a transmission line is then given as

$$
T_{L}=L_{O}+L_{C}
$$

That is,

$$
T_{L}=I^{2} R+242 \frac{(f+25)}{\delta} \cdot \sqrt[4]{\frac{r}{d}}\left(V-V_{c}\right)^{2} \cdot(10)^{-5} .
$$

The power losses are therefore given by

$$
T_{L}=R I^{2}+242 \frac{(f+25)}{\delta} \cdot \sqrt[4]{\frac{r}{d}}\left(V-V_{c}\right)^{2} \cdot(10)^{-5} \mathrm{~kW} .
$$

3.1. Predictive Model. It would have sufficed to substitute $V$ and $I$ from (11) and (12) into (17) so as to obtain an expression for the transmission losses but the transmission parameters $f, \delta, r$, and $d$ would have remained as undetermined factors. As such, it is more desirable to rewrite (17) implicitly in terms of the space variable $x$. Thus from (17),

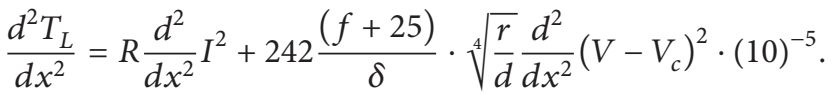


TABLe 2: Predicted power losses for a $330 \mathrm{kV}$ single circuit of the typical transmission network.

\begin{tabular}{|c|c|c|c|}
\hline $\begin{array}{l}\text { Length of } \\
\text { line in } \mathrm{km}\end{array}$ & $\begin{array}{l}\text { Power losses } \\
\text { (in MW) for } \\
\text { a load of } \\
100 \mathrm{MW} \\
\alpha=0.00005\end{array}$ & $\begin{array}{c}\text { Power losses } \\
\text { (in MW) for } \\
\text { a load of } \\
200 \mathrm{MW} \\
\alpha=0.00009\end{array}$ & $\begin{array}{c}\text { Power losses } \\
\text { (in MW) for } \\
\text { a load of } \\
300 \mathrm{MW} \\
\alpha=0.00014\end{array}$ \\
\hline 10 & 0.0500 & 0.1800 & 0.4300 \\
\hline 20 & 0.1000 & 0.3598 & 0.8594 \\
\hline 50 & 0.2500 & 0.8983 & 2.1438 \\
\hline 100 & 0.5000 & 1.7927 & 4.2724 \\
\hline 200 & 0.9953 & 3.5694 & 8.4839 \\
\hline 300 & 1.4892 & 5.3301 & 12.6354 \\
\hline
\end{tabular}

TABLE 3: Simulated results of power losses on $330 \mathrm{kV}$ single circuit of the Nigerian transmission network.

\begin{tabular}{lccc}
\hline $\begin{array}{l}\text { Length of } \\
\text { line in km }\end{array}$ & $\begin{array}{c}\text { Power losses } \\
\text { (in MW) for } \\
\text { a load of } \\
100 \mathrm{MW}\end{array}$ & $\begin{array}{c}\text { Power losses } \\
\text { (in MW) for } \\
\text { a load of } \\
200 \mathrm{MW}\end{array}$ & $\begin{array}{c}\text { Power losses } \\
\text { (in MW) for } \\
\text { a load of } \\
300 \mathrm{MW}\end{array}$ \\
\hline 10 & 0.05 & 0.18 & 0.43 \\
20 & 0.09 & 0.37 & 0.87 \\
40 & 0.18 & 0.73 & 1.75 \\
60 & 0.26 & 1.10 & 2.84 \\
100 & 0.41 & 1.85 & 4.66 \\
200 & 0.76 & 3.77 & 10.86 \\
300 & 1.10 & 5.85 & 24.40 \\
\hline
\end{tabular}

On integrating and utilising (2)-(9), (18) is simplified to

$$
\frac{d^{2} T_{L}}{d x^{2}}-2 R G(1+R G) T_{L}=0 .
$$

Solving (19) together with the requisite boundary conditions

$$
\begin{gathered}
T_{L}(0)=0, \\
T_{L}(l)=P_{0}, \quad l \longrightarrow \infty,
\end{gathered}
$$

where $P_{0}$ denotes the transmitted power. The solution to the transmission power losses model is obtained as

$$
T_{L}=P_{0}\left(1-e^{-\alpha x}\right)
$$

where $\alpha=\sqrt{2 R G(1+R G)}$.

Table 2 presents the predicted power losses for a $330 \mathrm{kV}$ single circuit of the Nigerian transmission network.

Table 3 presents the results obtained by Onohaebi and Odiase [21] using the power world simulator for loads of $100 \mathrm{MW}, 200 \mathrm{MW}$, and $300 \mathrm{MW}$ with the associated impedances for various lengths.

\section{Analysis and Discussion of Results}

Perusal of the results presented in Tables 1 and 2 confirms consistency in reduction of the numerical solutions returned, and so the results are in line with reality. On the other hand the results in Table 3 show close agreement with those in Table 2 . Hence, the predictions are in line with reality and empirical data.

The evolution of current and voltage on high tension transmission lines as well as the power losses when modelled evolved as second order ordinary differential equations. With appropriate boundary conditions, the solutions obtained are prescribed in closed forms. Allotting values to the input factors, numerical values were obtained for the requisite factors-current, voltage, and power losses.

An advantage of the analytic expressions obtained in this study is that numerical values can be computed with a handheld calculator unlike in the method of Král et al. [22] that requires the use of a software package.

Based on the above observations, the models can be used to predict requisite electrical measures along typical transmission lines. With these measures, electric transmissionrelated activities can be planned with a view to enhancing efficiency of the electric power system.

\section{Conclusion}

The equations describing the evolution of current and voltage along transmission lines have been utilised to fashion tools to predict requisite electrical measures such as current, voltage, and power losses.

The evolution of current and voltage on transmission lines is a process that can aid the determination of current and voltage as a function of the space variable $x$ on transmission lines. Fashioning the power loss function as a mathematical physics expression led to the formulation of a predictive model for power losses explicitly in terms of the space variable only.

\section{Conflict of Interests}

The authors declare that there is no conflict of interests regarding the publication of this paper.

\section{References}

[1] O. I. Okoro and E. Chikuni, "Power sector reforms in Nigeria: opportunities and challenges," Journal of Energy in Southern Africa, vol. 18, no. 3, pp. 52-57, 2007.

[2] A. P. Kenneth and A. T. John, "The benefit of distribution generation to the Nigerian electric utility system," Journal of Basic and Applied Scientific Research, vol. 3, no. 2, pp. 381-385, 2013.

[3] T. Smed, G. Andersson, G. B. Sheble, and L. L. Grigsby, "A new approach to AC/DC power flow," IEEE Transactions on Power Systems, vol. 6, no. 3, pp. 1238-1244, 1991.

[4] A. Sakhavati, M. Yaltagiani, S. S. Ahari, and S. S. Mahaei, "765 $\mathrm{kV}$ transmission line design (Electrical section)," International Journal of Electrical and Computer Engineering, vol. 2, no. 5, pp. 698-707, 2012.

[5] M. W. Gustafson and J. S. Baylor, "Transmission loss evaluation for electric systems," IEEE Transactions on Power Systems, vol. 3, no. 3, pp. 1026-1032, 1988. 
[6] L. Ramesh, S. P. Chowdhury, S. Chowdhury, A. A. Natarajan, and C. T. Gaunt, "Minimization of power loss in distribution networks by different techniques," International Journal of Electrical Power and Energy Systems Engineering, vol. 2, no. 1, pp. 1-6, 2009.

[7] N. Rugthaicharoencheep and S. Sirisumrannukul, "Feeder reconfiguration for lossreduction in distribution system with distributed generators by Tabu Search," GMSARN International Journal, vol. 3, pp. 47-54, 2009.

[8] N. Sinsuphun, U. Leeton, U. Kwannetr, D. Uthitsunthorn, and T. Kulworawanichpong, "Loss minimization using optimal power flow based on swarm intelligences," Transactions on Electrical Engineering, Electronics, and Communications, vol. 9, no. 1, pp. 212-222, 2011.

[9] O. M. Bamigbola, M. M. Ali, and M. O. Oke, "Mathematical modeling of electric power flow and the minimization of power losses on power transmission lines," submitted.

[10] H. Ambriz-Pérez, E. Acha, and C. R. Fuerte-Esquivel, "High voltage direct current modelling in optimal power flows," International Journal of Electrical Power and Energy Systems, vol. 30, no. 3, pp. 157-168, 2008.

[11] T. Dhaene and D. De Zutter, "Selection of lumped element models for coupled lossy transmission lines," IEEE Transactions on Computer-Aided Design of Integrated Circuits and Systems, vol. 11, no. 7, pp. 805-815, 1992.

[12] J. D. Glover, M. S. Sarma, and T. J. Overby, Power System and Design, Cengage Learning, Stamford, Conn, USA, 5th edition, 2008.

[13] C. R. Paul, Fundamentals of Electric Circuit Analysis, John Wiley, 2001.

[14] V. Král, S. Rusek, and L. Rudolf, "Calculation and estimation of technical losses in transmission networks," Electrical Review, vol. 88, no. 8, pp. 88-91, 2012.

[15] I. Sarajcev, M. Majstrovic, and I. Medic, "Calculation of losses in electric power cables as the base for cable temperature analysis," Journal of Advanced Computational Methods in Heat Transfer, vol. 4, pp. 529-537, 2003.

[16] M. H. Hesse, "Electromagnetic and electrostatic transmission line parameters by digital computer," IEEE Transactions on Power Apparatus and Systems, vol. 82, pp. 282-291, 1963.

[17] M. Abdel-Salam, Electrical Breakdown of Gases in High-Voltage Engineering: Theory and Practice, Marcel Dekker, New York, NY, USA, 2000, edited by, M.E. Khalifa.

[18] A. M. James, Electric Power System Applications of Optimization, McGraw-Hill, 2005.

[19] M. P. Bahrman and B. K. Johnson, "The ABCs of HVDC transmission technologies," IEEE Power and Energy Magazine, vol. 5, no. 2, pp. 32-44, 2007.

[20] C. R. Bayliss and B. J. Hardy, Transmission and Distribution Electrical Engineering, Elsevier, Amsterdam, The Netherlands, 2007.

[21] O. S. Onohaebi and O. F. Odiase, "Empirical modelling of power losses as a function of line loadings and lengths in the Nigeria $330 \mathrm{kV}$ transmission lines," International Journal of Academic Research, vol. 2, no. 3, pp. 47-53, 2010.

[22] V. Král, S. Rusek, and L. Rudolf, "Software for calculation of technical losses in transmission network," Przeglad Elektrotechniczny, vol. 87, no. 2, pp. 91-93, 2011. 


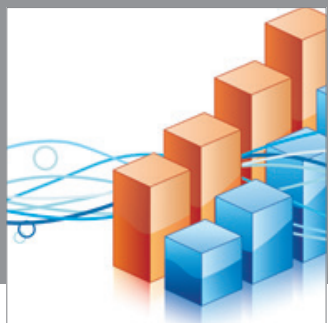

Advances in

Operations Research

mansans

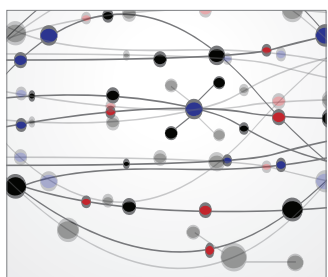

The Scientific World Journal
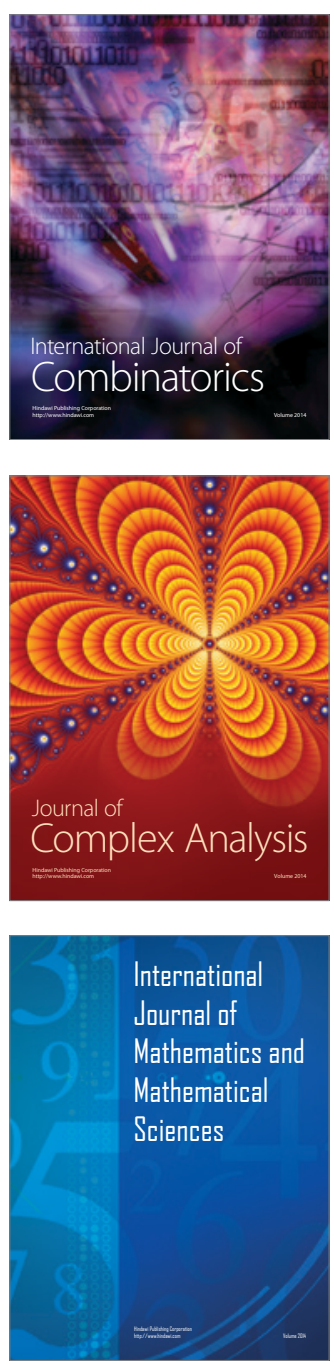
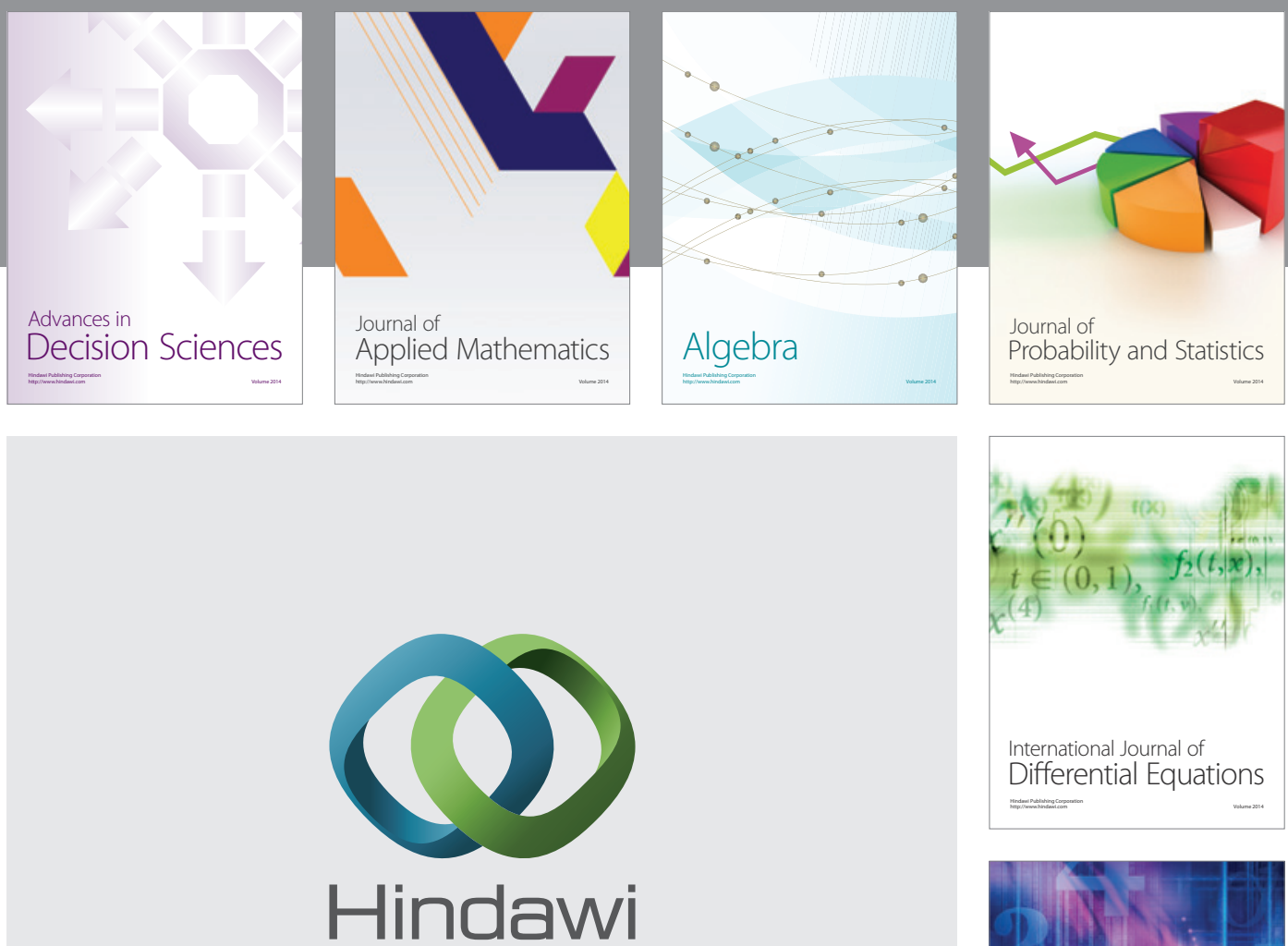

Submit your manuscripts at http://www.hindawi.com
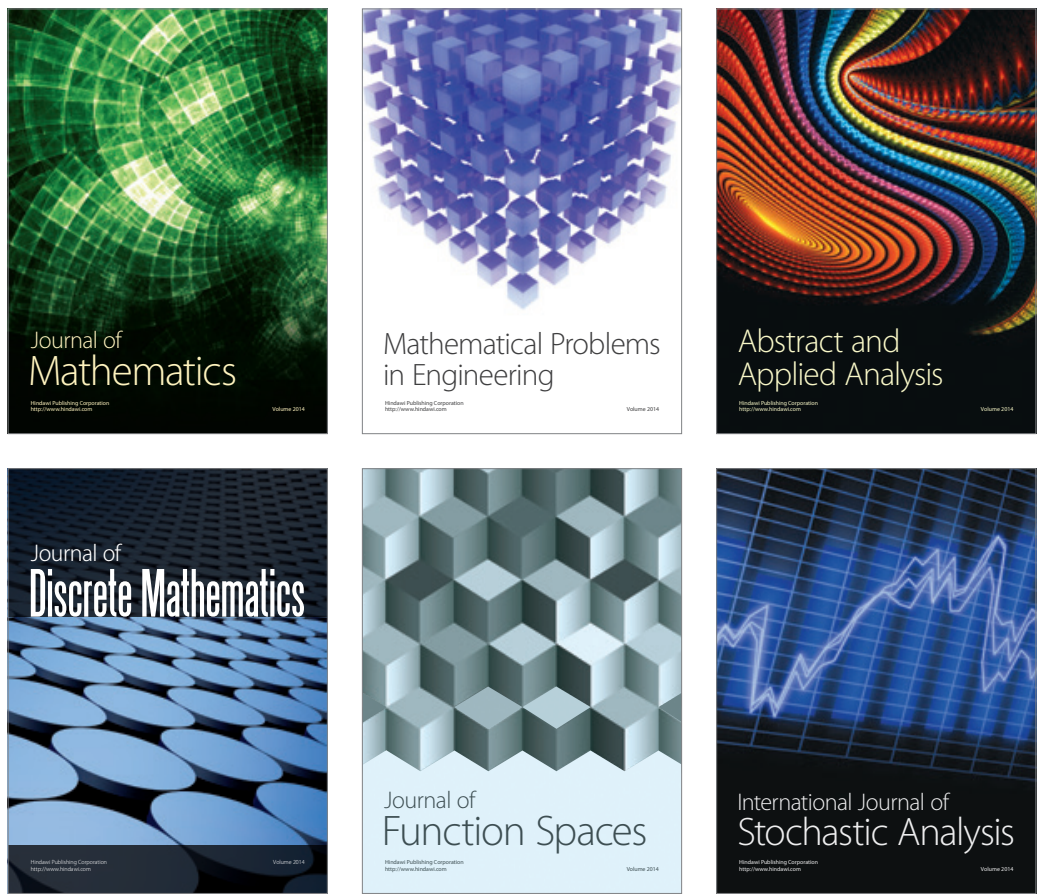

Journal of

Function Spaces

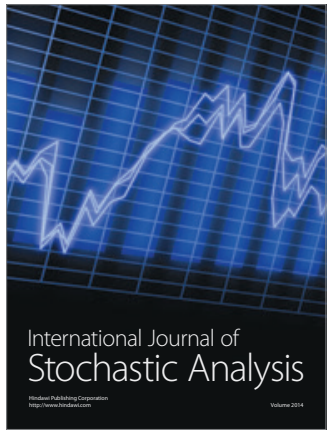

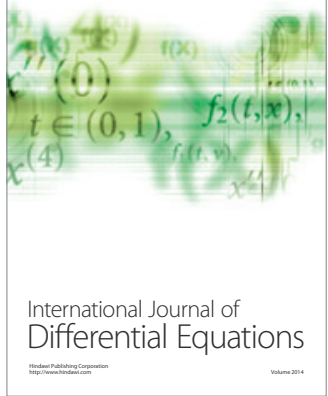
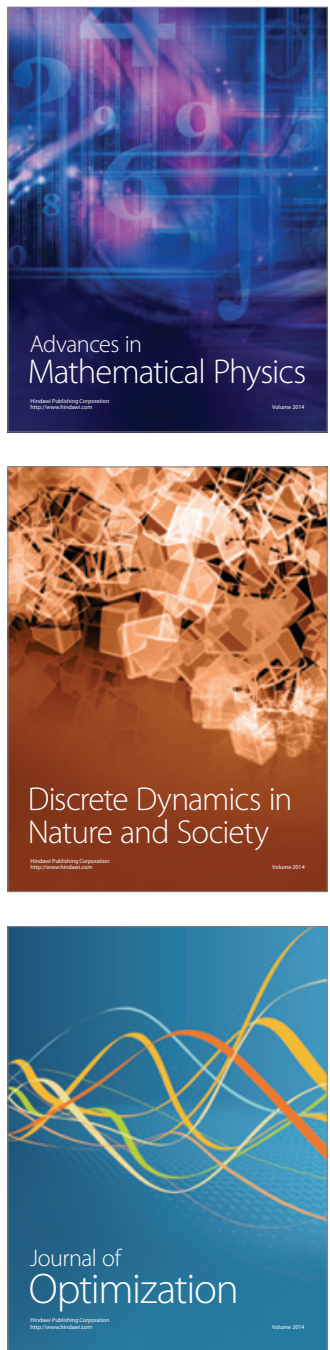